\title{
Adequação da rotulagem de alimentos industrializados para portadores de alergia alimentar
}

\author{
Adjustment of industrialized food labeling for food allergy sufferers \\ Adecuación del etiquetado de los alimentos industrializados para las personas alérgicas a \\ los alimentos
}

Larissa Perroni Bustamante Pinto ${ }^{1 *}$, Patrícia da Graça Leite Speridião.

\section{RESUMO}

Objetivo: Verificar a adequação e inadequação da rotulagem de alimentos industrializados destinados às crianças com idade entre seis meses e três anos, conforme a legislação vigente para alérgicos alimentares. Métodos: Trata-se de um estudo observacional e descritivo que incluiu, aleatoriamente, 67 alimentos industrializados, categorizados em lácteos, cereais, papas infantis/mucilagens e bebidas infantis. A coleta de dados contou com formulário tipo check list para a classificação em adequados ou inadequados, segundo as advertências e requisitos estabelecidos na legislação. Resultados: Dos 67 alimentos avaliados, $7(10,4 \%)$ deles apresentavam algum tipo de irregularidade na rotulagem. Das 4 categorias, os cereais e as bebidas apresentaram $100 \%$ de adequação dos rótulos em relação à legislação vigente para alérgicos. Os alimentos lácteos apresentaram $95,4 \%$ de rótulos adequados e $4,5 \%$ de inadequados, enquanto as papas infantis/mucilagens apresentaram $58,3 \%$ de adequação e $46,1 \%$ de inadequação, pela ausência de advertência e pelos requisitos obrigatórios. Conclusão: Alimentos lácteos e as papas infantis/mucilagens apresentaram rotulagem inadequada à legislação vigente o que pode acarretar prejuízo à saúde das crianças alérgicas e colocar em risco a segurança alimentar e nutricional desse grupo vulnerável.

Palavras-chave: Rotulagem de alimentos, Alérgenos, Alimentos infantis, Segurança alimentar.

\begin{abstract}
Objective: To verify the adequacy and inadequacy of the labeling of processed foods for children aged between six months and three years, according to the current legislation for food allergies. Methods: This is an observational and descriptive study that randomly included 67 processed foods, categorized into dairy products, cereals, baby foods/mucilages and drinks infantile. Data collection included a check list form for the classification as adequate or inadequate, according to the warnings and requirements established in the legislation. Results: Of the 67 foods evaluated, 7 (10.4\%) of them had some kind of irregularity in the labeling. Of the 4 categories, cereals and beverages showed $100 \%$ adequacy of labels in relation to current legislation for allergy sufferers. Dairy foods showed $95.4 \%$ of adequate labels and $4.5 \%$ of inadequate, while baby foods/mucilages presented $58.3 \%$ of adequacy and $46.1 \%$ of inadequacy, due to the absence of warning and mandatory requirements. Conclusion: Dairy foods and infant / mucilage porridge presented inadequate labeling to the current legislation, which can cause damage to the health of allergic children and put at risk of food and nutritional security of this vulnerable group.
\end{abstract}

Keywords: Food labeling, Allergens, Infant foods, Food securtiy.

\section{RESUMEN}

Objetivo: Verificar la adecuación e inadecuación del etiquetado de alimentos procesados para niños de entre seis meses y tres años, según la legislación vigente en alergias alimentarias. Métodos: Se trata de un

${ }^{1}$ Universidade Federal de São Paulo (UNIFESP), Santos - SP. *E-mail: larissapbustamante@yahoo.com.br

PUBLICADO EM: 9/2021 
estudio observacional y descriptivo que incluyó aleatoriamente 67 alimentos procesados, categorizados en lácteos, cereales, papillas/mucílagos y bebidas infantiles. La recolección de datos incluyó un formulario de verificación para la clasificación en adecuados o inadecuados, de acuerdo con las advertencias y requisitos establecidos. Resultados: Los 67 alimentos evaluados, 7(10,4\%) de ellos presentaban algún tipo de irregularidad en el etiquetado. De las 4 categorías, los cereales y las bebidas mostraron un 100\% de adecuación de las etiquetas en relación con la legislación vigente para las personas alérgicas. Los lácteos presentaron $95,4 \%$ de etiquetas adecuadas y $4,5 \%$ inadecuadas, mientras que las papillas/mucílagos infantiles presentaron $58,3 \%$ de adecuación y $46,1 \%$ de inadecuación, por la ausencia de advertencias y requisitos obligatorios. Conclusión: Alimentos lácteos y la papilla infantil/mucílago presentaron un etiquetado inadecuado a la legislación vigente, lo que puede causar daños a la salud de los niños alérgicos y poner en riesgo de seguridad alimentaria y nutricional de este grupo vulnerable.

Palabras clave: Etiquetado de alimentos, Alérgenos, Alimentos infantiles, Seguridad alimentaria.

\section{INTRODUÇÃO}

A alergia alimentar é caracterizada por reações adversas a determinados alimentos e depende de mecanismos imunológicos, mediados por anticorpos ou células, ou mistos, capazes de causar alterações funcionais em órgãos alvo (BERZUINO MB, et al., 2017; SPERIDIÃO PGL e MORAIS MB, 2014). É importante destacar que muitas crianças apresentam alergia alimentar desencadeada por mecanismos mediados por $\mathrm{lgE}$, o que pode determinar sua sobrevida nos casos da ingestão acidental ou não do alérgeno alimentar (ASSOCIAÇÃO BRASILEIRA DE ALERGIA E IMUNOPATOLOGIA, 2012).

A etiologia da alergia alimentar não está completamente elucidada, porém, sabe-se que pode provocar efeitos deletérios e até mesmo fatais à saúde do indivíduo (BERZUINO MB, et al., 2017; MORAIS EJF, et al., 2018). Quanto às manifestações clínicas, vários sistemas podem ser acometidos, desde o respiratório, provocando tosse, coriza e asma; passando pela pele, causando coceiras, eczemas e erupção e no trato digestório, manifestando dor abdominal, diarreia e náuseas ou até mesmo, anafilaxia (BERZUINO MB, et al., 2017; RAMOS REM, et al., 2013). Entre os sintomas mais comuns da alergia alimentar em crianças, destacam-se os abdominais devido à imaturidade da mucosa intestinal que permite maior absorção de proteínas, o que estimula a resposta imune e desencadeia o processo alérgico (SOLÉ D, et al., 2018).

É considerada problema de saúde pública, haja vista o aumento da sua prevalência, possivelmente, por consequentes mudanças no estilo de vida e nos hábitos alimentares atuais, como por exemplo, maior exposição às substâncias alérgicas presentes em alimentos processados, ultraprocessados e transgênicos disponíveis, acessíveis e consumidos. A alergia alimentar gera impacto relevante na saúde desses indivíduos vulneráveis, sendo o público infantil o mais acometido, pois, a indústria alimentícia dá atenção especial a esse nicho de mercado atraente, haja vista a necessidade dos pais e familiares em buscar alternativas que facilitem a alimentação diária das crianças (SOLÉ D, et al., 2018; MORAIS EJF, et al., 2018; POMIECINSKI F, et al., 2017; SILVA MBL, et al., 2017).

No Brasil, estima-se que as reações alérgicas de causa alimentar acometem de 6 a $8 \%$ das crianças com menos de 3 anos de idade e 2 a $3 \%$ dos adultos, sendo que em crianças até dois anos de idade, a alergia às proteínas do leite de vaca, é mais frequente (BERZUINO MB, et al., 2017; POMIECINSKI F, et al., 2017).

O tratamento da alergia alimentar se baseia na retirada ou exclusão total do alérgeno alimentar reconhecido ou possivelmente envolvido na sintomatologia, inclusive os produtos derivados e preparações que o contenham, visto que podem estar relacionados à patogênese da alergia alimentar (SOLÉ $D$, et al., 2018; SICHERER SH e SAMPSON HA, 2014). O sucesso do tratamento está diretamente relacionado à exclusão adequada do alérgeno e à habilidade dos pais e/ou familiares em realizar uma inspeção detalhada e precisa com leitura minuciosa dos rótulos dos alimentos a serem consumidos por crianças e que podem conter alérgenos (SOLÉ D, et al., 2018). 
Por meio da rotulagem, é possível ao consumidor, acesso à informação correta sobre as características dos alimentos que escolhe e, na ausência de informação adequada, o consumidor pode ter problemas graves de sua saúde, em especial os alérgicos (SILVA VCP, et al., 2014; REIS VS et al., 2020). Frente ao aumento da prevalência da alergia alimentar e da necessidade de realização da dieta de exclusão do suposto alérgeno alimentar, é importante que a população conheça o que consome, a fim de evitar complicações provenientes do consumo de alimentos inadequados, principalmente, pela ausência de informações pertinentes na rotulagem, as quais são extremamente necessárias para essa população (MARINS BR E JACOB SC, 2015).

Nesse sentido, o sucesso do tratamento da alergia alimentar depende, também, da informação responsável e adequada que pode ser encontrada nos rótulos dos alimentos industrializados para que os pacientes e seus familiares e responsáveis consigam manter a dieta livre dos componentes alergênicos Sendo assim, diante do cenário da alergia alimentar e dos possíveis riscos à saúde, é imperativo que se destaquem informações acerca da presença do alérgeno nos rótulos dos alimentos industrializados (RAMOS REM, et al., 2013).

É importante que exista regulamentação da rotulagem de alimentos com destaque para os alérgenos aos portadores de alergia alimentar, haja vista a necessidade de estarem seguros de que a dieta esteja livre desses alérgenos para preservar a saúde, garantindo o direito à informação e à aquisição de um produto com conhecimento de causa (CHADDAD MCC, 2014). ANPortanto, cabe à indústria o comprometimento de emitir informações corretas sobre a presença dos alérgenos alimentares nos rótulos dos produtos e, aos órgãos sanitários, a elaboração de legislação pertinente ao tema, com fiscalização contínua, prezando pelo bem-estar, saúde e qualidade de vida desses indivíduos (BERZUINO MB, et al., 2017).

Após intensa manifestação da sociedade sobre a relevância e a necessidade do direito à informação clara, precisa e objetiva, além da rotulagem que destaque os alérgenos alimentares, surgiu em 2 de julho de 2015, a Resolução de Diretoria Colegiada (RDC) oㅜ 26/2015 da Agência Nacional de Vigilância Sanitária (ANVISA) que estabelece os requisitos para rotulagem obrigatória dos alimentos causadores de alergias alimentares, sendo que se aplicando-se aos alimentos (incluindo as bebidas, ingredientes, aditivos alimentares e coadjuvantes de tecnologia) embalados fora da presença do consumidor (BERZUINO MB, et al., 2017; ANVISA, 2015).

Essa resolução foi elaborada considerando a alta prevalência de indivíduos portadores de alergias alimentares e com o objetivo de garantir não somente aos consumidores, mas também, aos seus familiares e cuidadores, o acesso às informações claras e precisas, em locais de fácil localização a fim de que a presença dos principais alimentos alergênicos possa ter o destaque adequado, impactando, desta forma, na escolha dos alimentos. Portanto, incentivar o hábito da leitura dos rótulos é uma forma de educar os consumidores a garantir seus direitos (ANVISA, 2015; MARINS BR e JACOB SC, 2015).

O acesso à alimentação e nutrição são elementos básicos para a promoção e proteção da saúde, possibilitando pleno potencial de crescimento e desenvolvimento humano, com qualidade de vida e cidadania, garantindo o direito à Segurança Alimentar e Nutricional (SAN) aos indivíduos, evitando que os mesmos estejam em situação de vulnerabilidade (SANTOS HF, et al., 2013).

Assim, diante desse cenário, pessoas com alergia alimentar demandam atenção especial no que se refere ao sistema de proteção à saúde e à informação sobre a presença intencional ou não de alérgenos alimentares nos rótulos dos produtos, visto que as manifestações clínicas podem ser deletérias (CHADDAD MCC, 2014).

Para tanto, considerando a importância da rotulagem obrigatória sobre a presença de alérgenos alimentares, o presente estudo objetivou verificar a adequação e inadequação da rotulagem de alimentos industrializados destinados às crianças com idade entre seis meses e três anos, confrontando-a com a legislação vigente para alérgicos - RDC no 26/2015. 


\section{MÉTODOS}

Trata-se de um estudo observacional e descritivo que incluiu, aleatoriamente, 67 alimentos industrializados, habitualmente consumidos (AIRES APP, et al., 2011; ZVEIBRÜCKER FP e MIRAGLIA F, 2012) por crianças com idade entre 6 meses e três anos. Esses alimentos foram classificados em 4 categorias: 1) lácteos; 2) cereais; 3) papas infantis/mucilagens e 4) bebidas (sucos de frutas). $O$ estudo foi realizado em três lojas de uma grande rede de supermercados.

Para verificar a adequação ou inadequação da rotulagem dos produtos em relação à legislação vigente, elaborou-se um instrumento tipo check list, dividido em duas partes, sendo que a primeira parte incluiu as advertências intencionais (Advertência 1: Alérgicos: Contém (nome alimento que causa alergia); Advertência 2: Alérgicos: Contém derivados de (nome alimento que causa alergia); Advertência 3: Alérgicos: Contém (nome alimento que causa alergia) e derivados) ou seja, a presença do alérgeno e/ou de seus derivados. A segunda parte contou com os requisitos previstos na legislação específica, sendo: 1) letras em caixa alta, 2) negrito, 3) com altura mínima de $2 \mathrm{~mm}$ e 4) cor contrastante com o fundo do rótulo, segundo a legislação (ANVISA, 2015).

Todos os alimentos avaliados foram considerados adequados quando contavam com a presença dos quatro requisitos previstos na legislação e, pelo menos, uma advertência intencional. A inadequação foi observada quando havia ausência de um ou mais requisitos previstos na legislação, além da ausência de advertências intencionais. Os resultados foram expressos em frequência absoluta e relativa.

O estudo foi aprovado pelo Comitê de Ética em Pesquisa (CEP) da Universidade Federal de São Paulo, sob no 8148270417.

\section{RESULTADOS}

Dos 67 produtos industrializados incluídos no estudo, a rotulagem de $7(10,4 \%)$ deles apresentou algum tipo de irregularidade em relação à legislação vigente, sendo considerados não adequados. Desses, 2 categorias de alimentos não atendiam à legislação quanto aos requisitos nem às advertências, sendo um leite integral UHT e uma papa de frutas. Esses produtos não continham informação alguma sobre a presença de alérgenos alimentares em seus rótulos, apenas apresentavam a lista de ingredientes. Os outros 5 alimentos (categoria de papas infantis/mucilagens) não apresentavam, se quer, uma das três advertências contempladas na resolução, contudo, atendiam aos requisitos (Figura 1).

Figura 1 - Percentual de adequação e não adequação da rotulagem dos alimentos industrializados de acordo com a RDC 26/2015.

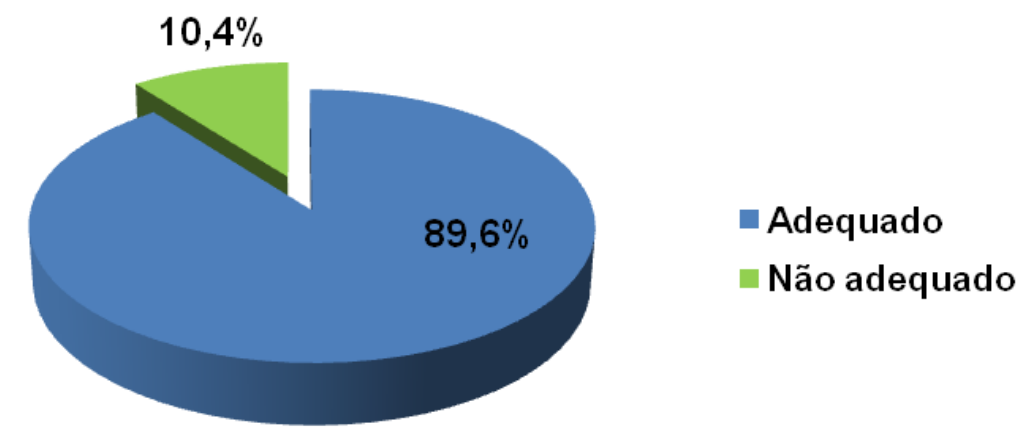

Fonte: Pinto LPB e Speridião PGL, 2021.

A maioria dos rótulos analisados, $62(92,5 \%)$ apresentou, no mínimo, uma advertência sobre a presença do alérgeno alimentar e foram considerados, adequados. Das 4 categorias de alimentos industrializados 
avaliados, os lácteos apresentaram 95,4\% (21) dos rótulos adequados à legislação, enquanto as categorias cereais e bebidas, apresentaram 100\% (27 e 5, respectivamente) de adequação em relação à RDC no 26/2015. Já a categoria papas infantis/mucilagens apresentou rotulagem com a maior frequência de inadequação, tanto pela ausência das advertências, quanto dos requisitos obrigatórios, representando 46,1\% (6) (Tabela 1).

Tabela 1 - Frequência absoluta, relativa da rotulagem adequação e inadequada por categorias de alimentos avaliados nos supermercados.

\begin{tabular}{lcccc}
\hline & \multicolumn{2}{c}{ Rótulos Adequados } & \multicolumn{2}{c}{ Rótulos Inadequados } \\
\cline { 2 - 5 } Categoria do alimento & $\mathbf{n}$ & $\%$ & $\mathbf{n}$ & $\%$ \\
\hline Lácteos $(\mathbf{n}=\mathbf{2 2})$ & 21 & $(95,4)$ & 1 & $(4,5)$ \\
Cereais $(\mathbf{n}=\mathbf{2 7})$ & 27 & $(100,0)$ & 0 & $(0,0)$ \\
Papas infantis/mucilagens $(\mathbf{n}=\mathbf{1 3})$ & 7 & $(58,3)$ & 6 & $(46,1)$ \\
Bebidas $(\mathbf{n}=\mathbf{5})$ & 5 & $(100,0)$ & 0 & $(0,0)$
\end{tabular}

Fonte: Pinto LPB e Speridião PGL, 2021.

Quanto à presença do tipo de alérgeno alimentar destacado no rótulo, leite esteve presente em 27 rótulos; soja em 26; trigo em 18; cevada em 11; aveia em 7 e, ovos em 5, para todas as categorias de alimentos (Figura 2).

Figura 2 - Frequência absoluta dos alérgenos nos rótulos dos alimentos avaliados nos supermercados.

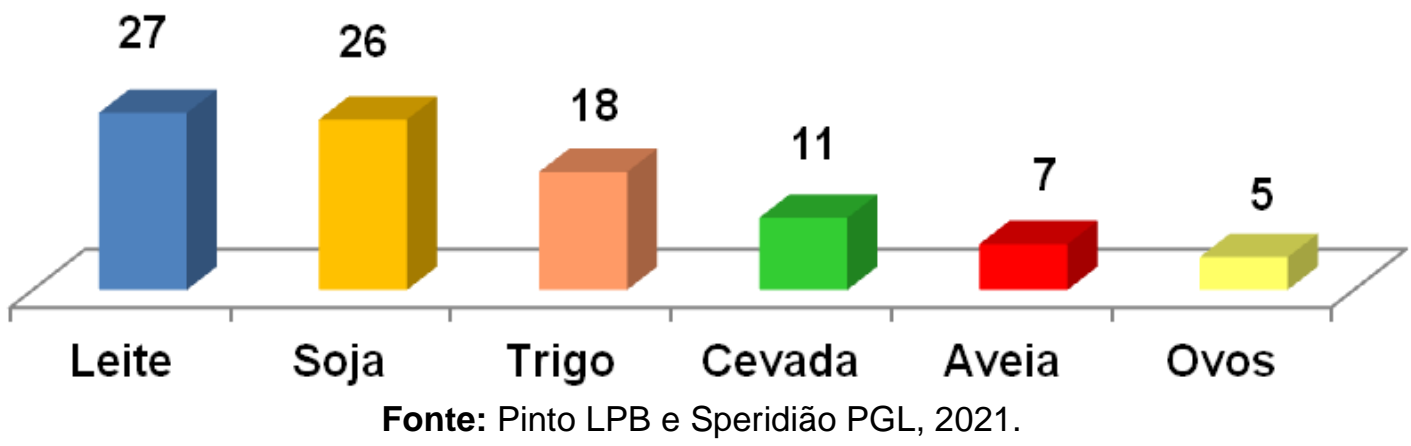

A expressão "pode conter" que se refere à possibilidade de contaminação cruzada, ou seja, quando o produto pode apresentar traços do alérgeno alimentar durante seu processo de fabricação, esteve ausente em $22(32,8 \%)$ dos rótulos avaliados. Entre as diferentes categorias de alimentos avaliados, os cereais apresentaram a maior frequência para essa expressão "pode conter". Os alérgenos alimentares que mais se associaram à expressão "pode conter" foram o trigo, aveia, centeio, cevada e o leite.

\section{DISCUSSÃO}

A Resolução da Diretoria Colegiada $n^{\circ}$ 26/2015, que "dispõe sobre os requisitos para rotulagem obrigatória dos principais alimentos que causam alergias alimentares" determina que as indústrias alimentícias informem a presença de alimentos alergênicos nos rótulos de seus produtos, sendo, portanto, a base a normatização da rotulagem a ser cumprida no Brasil. 
Em estudo no qual foram avaliados 102 rótulos de alimentos infantis, observou-se que 10,7\% não se encontravam em conformidade com a legislação para alérgicos conforme a RDC 26/2015, mesmo após o período de 12 meses, estabelecido para as indústrias se adequarem (ANVISA, 2015). Entre os rótulos avaliados, destacaram-se os biscoitos que são amplamente consumidos por crianças (MALLET ACT, et al., 2017).

Em relação aos alimentos avaliados em nosso estudo, foi possível observar que a grande maioria se encontrava adequada à legislação vigente para alérgicos, porém, em 7 do total de rótulos analisados, foi observado algum tipo de irregularidade em relação à legislação vigente, sendo, portanto, considerados não adequados para o consumo. Sob essa análise, os resultados obtidos neste trabalho são semelhantes aos encontrados por Mallet ACT, et al (2017) e, alertam para a necessidade de intervenção por parte do poder público, haja vista que os direitos dos consumidores com alergia alimentar, não estão sendo respeitados, decorridos seis anos da publicização da RDC 26/2015.

Em outro estudo conduzido por Rodrigues AKF, et al (2021) no qual se avaliou a conformidade de 60 rótulos de diferentes grupos de alimentos, encontrou-se um grande número de não conformidade relativa à ausência de declaração para alergênicos, mesmo após o prazo estipulado pelos órgãos sanitários. Da mesma forma, nossos resultados também evidenciaram essa não conformidade com a legislação vigente, reforçando a importância da necessidade de fiscalização constante do comércio de alimentos.

Um estudo realizado com 52 rótulos de alimentos mostrou que 19,2\% encontravam-se em conformidade com a legislação contra $80,8 \%$ que se encontravam em não conformidade. Em relação aos alimentos em não conformidade com a legislação, destacaram-se os leites, biscoitos e alimentos infantis (SMITH ACL e ALMEIDA-MURADIAN LB, 2011). Contudo, vale ressaltar que esse estudo foi realizado em 2011, período em que não havia legislação voltada para grupos vulneráveis, como o caso dos alérgicos. Entretanto, o estudo destacou inadequações em relação à rotulagem nutricional, o que poderia comprometer a segurança alimentar e nutricional dos indivíduos.

Ao se comparar os resultados do presente estudo com os resultados do estudo realizado em 2011 , pode-se evidenciar que mesmo havendo legislações próprias e tendo decorrido uma década, ainda existe a necessidade de adequação e conscientização por parte das indústrias alimentícias, além de efetivas ações de fiscalização por parte dos órgãos governamentais.

Quando analisados os resultados encontrados sobre a categoria dos produtos lácteos, observa-se que 95,4\% desses alimentos encontravam-se em adequação à legislação vigente. Resultado semelhante também foi encontrado no estudo de Soares NR e Nunes TP (2021) que encontrou entre os 100 rótulos analisados, apenas $1 \mathrm{com}$ ausência na declaração de alergênicos. É possível aventar que esses resultados se devem ao fato de que essa categoria de alimentos é amplamente consumida por crianças, tornando-a uma opção de grande destaque entre os adultos responsáveis por crianças e, assim, devem apresentar rotulagem a mais adequada possível à legislação para evitar maiores problemas. Porém, ainda é possível encontrar ausência de declaração para alergênicos conforme mostra o estudo de Farias et al (2017) que analisou rótulos das embalagens de produtos derivados do leite.

Em estudo conduzido por Silva MBL, et al. (2017) no qual se avaliou a conformidade da rotulagem de diversos alimentos infantis, encontrou-se não conformidade em relação à rotulagem nutricional de sopas, papinhas e purês. Em outro estudo realizado por Brito LF, et al. (2016) também se observou inadequação na rotulagem de alimentos à base de cereais em 12 dos 30 rótulos analisados. Tais resultados se apresentam bastante semelhantes aos encontrados no presente estudo, pois, nota-se que a categoria de papas infantis/mucilagens apresentou a maior frequência de inadequação, tanto pela ausência das advertências, quanto dos requisitos obrigatórios.

Com relação às bebidas, todos os sucos à base de frutas apresentaram rótulos adequados, entretanto, o requisito "cor contrastante com o fundo do rótulo" não se apresentou totalmente, apropriado, o que pode contribuir para leitura equivocada. Porém, esse achado não influenciou a classificação da rotulagem como 
inadequada, mas, permite destacar que essa se encontra na contramão do que é proposto pelo Instituto Brasileiro de Defesa do Consumidor (IDEC) o qual recomenda que as embalagens de alimentos tenham fundo branco para garantir contraste suficiente à leitura dos itens (MINISTÉRIO DA SAÚDE, 2014).

Em Pelotas, um estudo conduzido por Cavada CS, et al. (2012) com 241 consumidores de supermercados, destacou que existe influência positiva da leitura de rótulos dos alimentos sobre a compra de alimentos, já que a rotulagem se caracteriza como um importante canal de comunicação entre 0 consumidor e o produto. Os autores apontam ainda que, se o rótulo é bem compreendido, esse permite escolhas alimentares mais criteriosas.

O fato de o presente estudo ter encontrado produtos não conformes com a RDC ํㅡ 26/2015, mesmo após o prazo de adequação corrobora com o estudo de Miranda e Gama (2018) que analisaram rótulos de produtos comercializados e encontraram $31,7 \%$ de rótulos inadequados. Tais resultados destacam a dificuldade das indústrias em se adequarem e a falta de fiscalização por parte do governo em implementar as normas vigentes para a presença de alimentos alergênicos.

As informações contidas nos rótulos podem auxiliar na prevenção e no tratamento de alergia alimentar sendo que os brasileiros contam atualmente, com uma resolução aliada ao Código de Defesa do Consumidor, a RDC № 26/2015 para fazer valer os seus direitos (ANVISA, 2016; ANVISA, 2015).

Outro aspecto importante destacado em nosso estudo se refere à expressão "Pode Conter", que não foi observada nos rótulos dos produtos lácteos, possivelmente, pelo fato de que seu processamento seja mais simples quando comparado às demais categorias de alimentos analisados. A expressão "Pode Conter" foi mais frequente nos alimentos ultraprocessados, revelando que quanto maior o grau de processamento do alimento, maior é a frequência de alérgenos presentes nele. Contudo, é importante ressaltar que esta expressão não consta como item obrigatório na RDC 26/2015 (ANVISA, 2015). Observou-se também que quanto mais processado ou ultraprocessado é o alimento, maior é a possibilidade de contaminação cruzada por alérgenos alimentares. Um exemplo dessa situação é o macarrão instantâneo que continha em seu rótulo a descrição de 16 alérgenos alimentares antecedidos da expressão "pode conter".

Destaque deve ser dado ao Guia Alimentar para a População Brasileira que reforça a necessidade de aumentar o consumo de alimentos in natura e reduzir o consumo de alimentos processados e ultraprocessados para garantir a prática de alimentação saudável e segura (MINISTÉRIO DA SAÚDE, 2014). No caso das crianças alérgicas, é recomendável evitar o consumo de alimentos industrializados, haja vista que quanto maior é grau de processamento do alimento, mais alergênico ele é, sendo menos saudável e menos seguro.

Por fim, é válido destacar a importância da promulgação da RDC nำ26/2015, que estabeleceu requisitos e advertências para a presença de alérgenos alimentares, constituindo-se em uma normativa atual e de grande relevância para a sociedade, principalmente ao grupo de pessoas e lou familiares com alergia alimentar com o objetivo de garantir uma melhor qualidade de vida a esses indivíduos. Mas, para que isso possa realmente ser garantido, é necessário o comprometimento da indústria para que possa cumprir a determinação legal e uma maior fiscalização nos mercados consumidores e, evidente uma maior participação da sociedade em fazer cumprir seus direitos, como o acesso à informação e à rotulagem para os alimentos com ingredientes alergênicos.

\section{CONCLUSÃO}

Diante dos resultados encontrados é importante ressaltar que o presente estudo vem contribuir de forma contundente com o cenário nacional regulatório para rótulos, além de proporcionar informação e conhecimento atualizado sobre a situação da rotulagem de algumas categorias de alimentos industrializados, comumente, consumidos por crianças com alergia alimentar. Portanto, faz-se necessário destacar a importância do acesso dos consumidores a informações claras e precisas contidas nos rótulos e que possa haver uma fiscalização mais efetiva para que o direito dos alérgicos seja garantido e que assim tenham uma melhor qualidade de vida. 


\section{REFERÊNCIAS}

1. ASSOCIAÇÃO BRASILEIRA DE ALERGIA E IMUNOPATOLOGIA. Guia prático de diagnóstico e tratamento da Alergia às Proteínas do Leite de Vaca mediada pela imunoglobulina E. Revista Brasileira de Alergia e Imunopatologia, 2012; 35(6): 203-33.

2. AGÊNCIA NACIONAL DE VIGILÂNCIA SANITÁRIA (ANVISA). Perguntas e Respostas sobre Rotulagem de Alimentos Alergênicos. Ministério da Saúde. Brasil, 2016. Disponível em http://segurancaalimentar.mprs.mp.br/alergenicos/Perguntas-e-Respostas.pdf. Acesso em: 14 de novembro de 2017.

3. AGÊNCIA NACIONAL DE VIGILÂNCIA SANITÁRIA (ANVISA Resolução RDC n 26, de 02 de julho de 2015. Dispõe sobre os requisitos para rotulagem obrigatória dos principais alimentos que causam alergias alimentares. Ministério da Saúde. Brasil, 2015. Disponível em: https://www.gov.br/agricultura/pt-br/assuntos/inspecao/produtosvegetal/legislacao-1/biblioteca-de-normas-vinhos-e-bebidas/resolucao-rdc-no-26-de-2-de-julho-de-2015.pdf/view. Acesso em: novembro de 2017.

4. BERZUINO MB, et al. Alergia alimentar e o cenário regulatório no Brasil. Rev. Eletr. Farm., 2017; 14(2):23-36.

5. BRITO LF, et al. Avaliação da rotulagem de alimentos à base de cereais para a alimentação de lactentes e crianças na primeira infância. Demetra; 2016;11(1): 111-120.

6. CAVADA GS, et al. Rotulagem nutricional: você sabe o que está comendo? Braz J Food Technol, 2012: 84-88.

7. CHADDAD MCC. Informação sobre a presença de alérgenos nos rótulos de alimentos: responsabilidade do Estado na garantia dos direitos à saúde e à alimentação adequada da população com alergia alimentar. Demetra, 2014; 9(1): 369-392.

8. FARIAS TAL, et al. Rotulagem de derivados lácteos frente às novas legislações da ANVISA. Revista Brasileira de Agrotecnologia, 2017; 7(1): 106-109.

9. MALLET ACT, et al. Adequação das rotulagens alimentícias frente à legislação vigente. Cadernos UniFOA, 2017; 35: 101-110.

10. MARINS BR, JACOB SC. Avaliação do hábito de leitura e da compreensão da rotulagem por consumidores de Niterói, RJ. Visa em Debate, 2015; 3(3): 122-129.

11. MINISTÉRIO DA SAÚDE. Secretaria de atenção à saúde. Departamento de atenção básica. Guia alimentar para a $\begin{array}{lllll}\text { população } & \text { brasileira. } & \text { Brasil, } & \text { Disponível }\end{array}$ https://bvsms.saude.gov.br/bvs/publicacoes/guia_alimentar_populacao_brasileira_2ed.pdf. Acesso em: novembro de 2017.

12. MIRANDA CCS, GAMA LLA. Inadequação da rotulagem de alergênicos: risco para indivíduos com hipersensibilidade alimentar. Demetra, 2018; 13(3):731-743.

13. MORAIS EJF, et al. Alimentos alergênicos e seus efeitos na saúde humana: uma breve revisão da literatura. International Journal of Nutrology 2018; 11(S01): S24-S327.

14. POMIECINSKI F, et al. Estamos vivendo uma epidemia de alergia alimentar? Rev. Bras Promoç Saúde, Fortaleza, $2017 ; 30(3): 1-3$.

15. RAMOS REM, et al. Alergia alimentar: reações e métodos diagnósticos. J Manag Prim Health Care, 2013; 4(2): 5463.

16. RODRIGUES AKF, et al. Análise da conformidade da rotulagem de diferentes grupos de alimentos comercializados na cidade de Fortaleza, CE. Journal of Medicine and Health Promotion. 2021; 6: 245-255.

17. SANTOS HF, et al. Análise de rotulagem nutricional de alimentos que compõem a cesta básica. Revista Nutrir Gerais, 2013; 7(13): 1030-1043.

18. SICHERER SH, SAMPSON HA. Food allergy: Epidemiology, pathogenesis, diagnosis, and treatment. J Allergy Clin Immunol, February 2014.

19. SILVA JA, NASCIMENTO BMS. Análise da adequação de rótulos de alimentos infantis com conteúdo alergênico segundo a RDC 26/2015. Resumo do Congresso Internacional de Atividade Física Nutrição e Saúde: 2017.

20. SILVA MBL, et al. Rotulagem de alimentos infantis à base de frutas, hortaliças e/ou cereais: uma análise de conformidade frente à legislação brasileira. J Health Sci, 2017; 19(1): 55-61.

21. SILVA VCP, et al. Avaliação das rotulagens e informações nutricionais dos pães integrais: fibras, sódio e adequação a legislação vigente. Demetra, 2014; 9(4): 985-1001.

22. SMITH ACL, ALMEIDA-MURADIAN LB. Rotulagem de alimentos: avaliação da conformidade frente à legislação e propostas para a sua melhoria. Revista Instituto Adolfo Lutz, 2011; 70(4): 463-72.

23. SOARES DJ, et al. Análise do comportamento dos consumidores com relação à compreensão e entendimento das informações dos rótulos de alimentos. Revista Agropecuária Técnica, 2016; 37(1): 105-111.

24. SOARES NR, NUNES TP. Avaliação da conformidade de rótulos de produtos lácteos frente à legislação vigente e a percepção do consumidor sobre rotulagem. Research, Society and Development, 2021;10(24110313223).

25. SOLE D, et al. Consenso Brasileiro sobre Alergia Alimentar: 2018 - Parte 2. Diagnóstico, tratamento e prevenção. Arq Asma Alerg Imunol. 2018; 2(1): 39-82

26. SPERIDIÃO PGL, MORAIS MB. Intolerância à lactose e alergia alimentar. In: Cuppari L. Guias de Nutrição: clínica no adulto. 3 ed. Barueri, SP: Manole, 2014.

27. ZVEIBRÜCKER FP, MIRAGLIA F. Avaliação do consumo alimentar de pré-escolares frequentadores de EMEls no município de Nova Santa Rita, RS. CIPPUS - Revista de Iniciação Científica do Unilasalle, 2012; 1(1). 